\title{
A STEM Parallel Diffraction Technique Applied to Nanomaterials
}

\author{
K.J. Ganesh*, M. Kawasaki**, J.P. Zhou*, P.J. Ferreira* \\ *Materials Science and Engineering, University of Texas at Austin, Austin, TX, 78712, USA \\ **JEOL, USA Inc., 11 Dearborn Rd., Peabody, MA 01960, USA
}

A technique for obtaining spot electron diffraction patterns from nanostructures as small as $3 \mathrm{~nm}$ has been developed in a JEOL 2010F TEM/STEM instrument operated at $200 \mathrm{kV}$ and equipped with an ultra high-resolution pole piece. This diffraction STEM technique (D-STEM) allows for an automated acquisition of spot diffraction patterns from ensembles of individual nanostructures, with enhanced levels of control and precision, a task currently challenging for modern instruments.

The D-STEM mode consists of a parallel probe scanned over the specimen to obtain a bright-field or dark-field STEM image. The beam is subsequently positioned on the image, at a particular point of interest, while the diffraction pattern is recorded by a CCD camera.

In the D-STEM mode, the Free-Lens control feature in the instrument has been explored to optimize the ray path (Fig.1a), such that well-defined spot diffraction patterns can be obtained. In terms of electron optics, the major distinction between a conventional STEM configuration (CSTEM) (Fig.1b) and D-STEM is the use of a strongly excited condenser mini-lens $(\mathrm{CM}=8.07 \mathrm{~V})$ in the D-STEM mode. This creates a demagnified point source of illumination close to the pre-field of the objective lens, resulting in a parallel beam on the specimen.

In general, the crossover image following the third condenser lens (C3) serves as the effective point source of illumination for the objective pre-field. Therefore, in the case of the C-STEM configuration, where the CM lens are deactivated, a higher crossover of the beam with respect to the objective pre-field results in a sharp convergent probe on the specimen, which should be avoided for producing spot diffraction patterns. On the other hand, in D-STEM mode, the CM lens, operated at maximum excitation, together with a stronger demagnification of the electron source (C1 lens), produces a parallel beam with 1-2 nm spot size. Furthermore, the smallest condenser aperture of $10 \mu \mathrm{m}$ is employed to obtain sharper maxima at the back focal plane of the objective lens.

Alignment in C-STEM is performed using a Ronchigram, while in D-STEM, the alignment procedure is essentially different. Initially, the specimen is placed at the eucentric height and a phase contrast image is taken using regular TEM illumination. Then, the operating mode is changed to STEM, keeping the objective lens strength $(6.85 \mathrm{~V})$ constant. In the STEM SPOT mode, the FreeLens control is activated and the Voltage-Center alignment is carried out to position the electron beam along the optical axis of the objective lens. Subsequently, STEM imaging is performed in the PIC mode and recorded using the DigiScan ${ }^{\circledR}$ detector. This image is used for positioning the beam accurately at the point of interest and projecting the back focal plane onto the CCD camera. Fig. 2a and Fig. 2c depict bright-field STEM images of silver and boron nitride nanoparticles of sizes $\sim 4 \mathrm{~nm}$ and $\sim 3 \mathrm{~nm}$, respectively. Fig. $2 \mathrm{~b}$ and Fig. $2 \mathrm{~d}$ show the spot diffraction patterns obtained from individual particles $\mathrm{P} 1$ and $\mathrm{P} 2$, respectively.

In summary, to set a D-STEM mode capable of acquiring spot diffraction patterns from nanostructures as small as $3 \mathrm{~nm}$, the instrument requires a STEM bright-field or dark-field detector, FasTEM $^{\circledR}$, Free-Lens control, a small condenser aperture, and a bottom mounted CCD camera. In this fashion, as the D-STEM technique can be automated (using Gatan ${ }^{\circledR}$ software "STEM-diffraction imaging"), spot diffraction patterns can be obtained from every pixel in the STEM image. 
$1 \mathrm{a}$

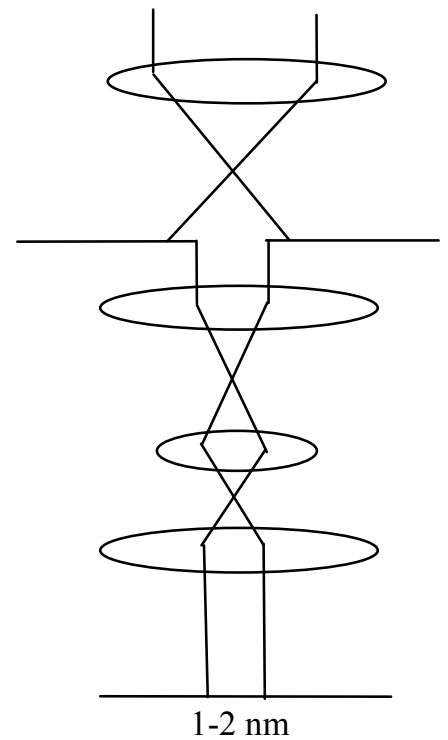

$\mathrm{C} 1=5.90$

$\mathrm{C} 2=10 \mu \mathrm{m}$

$\mathrm{C} 3=4.07$

$\mathrm{CM}=8.07$

Objective Pre-field

Specimen
$1 b$

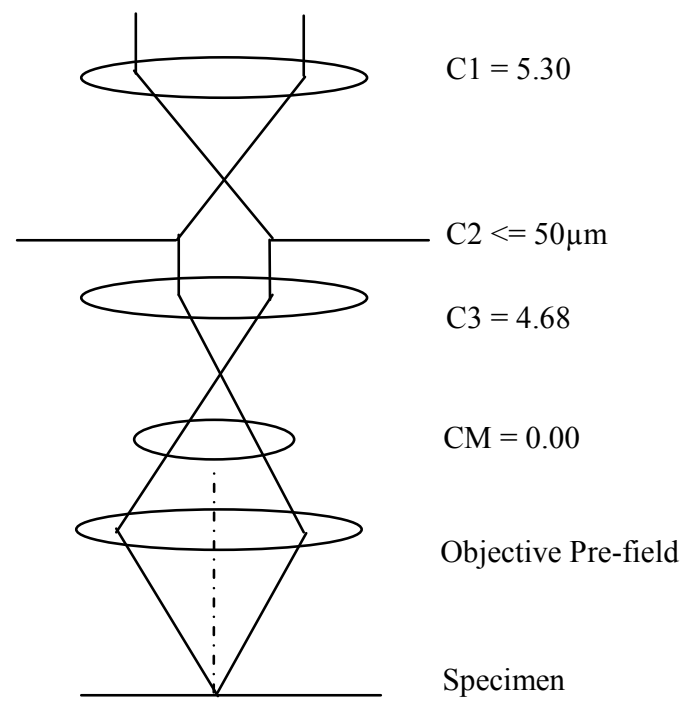

Fig. 1 Schematic ray diagram showing: a) D-STEM; b) C-STEM. The lens-strength is measured in Volts.
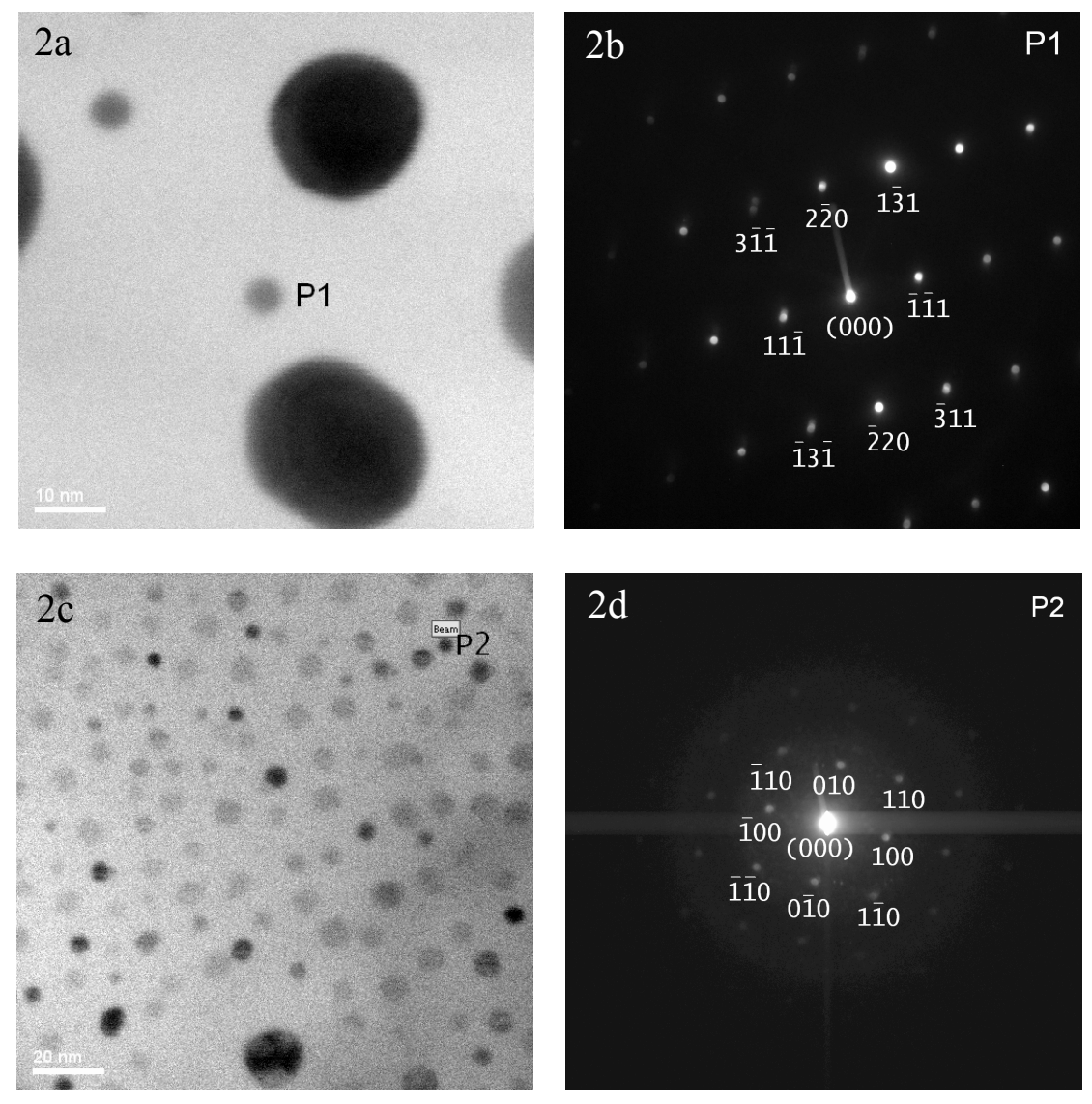

Fig. 2. a) Bright-field STEM image of silver nanoparticles; b) Diffraction pattern of P1 ( 4nm in size) along the [112] beam direction; c) Bright-field STEM image of Boron Nitride nanoparticles; d) Diffraction pattern of $\mathrm{P} 2(\sim 3 \mathrm{~nm}$ in size $)$ along the [001] beam direction. 